\title{
Fluorescent molecular imaging can improve intraoperative sentinel margin detection in oral squamous cell carcinoma
}

Giri Krishnan MBBS, MClinSc ${ }^{1,2}$, Nynke S. van den Berg $\mathrm{PhD}^{1}$, Naoki Nishio MD, PhD ${ }^{1,3}$, Shrey Kapoor MS ${ }^{1}$, Jaqueline Pei BS ${ }^{1}$, Laura Freeman MD $^{1}$, Yu-Jin Lee MD ${ }^{1}$, Stan van Keulen $\mathrm{MD}^{1}$, Shayan Farkurnejad MD${ }^{1}$, James Condon $\mathrm{MBBS}^{4}$, Fred M. Baik MD ${ }^{1}$, Brock A. Martin $\mathrm{MD}^{5}$, Eben L. Rosenthal MD ${ }^{1^{*}}$

\section{Affiliations:}

${ }^{1}$ Department of Otolaryngology-Head and Neck Surgery, Stanford University School of Medicine, Stanford, CA, United States;

${ }^{2}$ Department of Otolaryngology, Head and Neck Surgery, The University of Adelaide, Adelaide, SA, Australia;

${ }^{3}$ Department of Otorhinolaryngology, Nagoya University Graduate School of Medicine, Nagoya, Aichi, Japan.

${ }^{4}$ Department of Public Health, School of Medicine, The University of Adelaide, Adelaide, SA, Australia;

${ }^{5}$ Department of Pathology, Stanford University School of Medicine, Stanford, CA, United States;

\section{Funding sources:}

This work was supported in part by the Stanford Comprehensive Cancer Center, the Stanford University School of Medicine Medical Scholars Program, the Garnett Passe and Rodney William's Memorial Foundation, the Fulbright Association, the Netherlands Organization for Scientific Research (Rubicon; 019.171LW.022), the National Institutes of Health and the National Cancer Institute (R01CA190306), the Stanford Molecular Imaging Scholars (SMIS) program (T32CA118681), and an institutional equipment loan from LI-COR Biosciences, Inc.

\section{Disclaimer:}

E.L.R. has institutional equipment loans from LICOR Biosciences and Stryker. All other authors declare no conflict of interest.

\section{Corresponding Author:}

Eben L. Rosenthal, MD

Department of OHNS

900 Blake Wilbur Drive, Stanford, CA 94305

Tel: (650) 498-6000; Fax: (650) 724-1458

Email: elr@stanford.edu 


\section{First Author:}

Dr. Giri Krishnan MBBS, MClinSc

Department of Otolaryngology-Head and Neck Surgery,

The University of Adelaide

28 Woodville Rd, Woodville South SA 5011

Tel: (08) 8222 7158; Fax: (08) 82227419

Email: giri.krishnan@adelaide.edu.au

Manuscript word count: 4,928

Figures: 5

Tables: 2

Running title: Fluorescent sentinel margin detection

Immediate Open Access: Creative Commons Attribution 4.0 International License (CC BY) allows users to share and adapt with attribution, excluding materials credited to previous publications.

License: https://creativecommons.org/licenses/by/4.0/. Details: https://jnm.snmjournals.org/page/permissions.

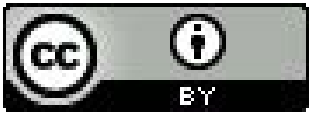




\begin{abstract}
Rationale:

In head and neck cancer, a major limitation of current intraoperative margin analysis is the ability to detect areas most likely to be positive based on specimen palpation, especially for larger specimens where sampling error limits detection of positive margins. This study aims to prospectively examine the clinical value of fluorescent molecular imaging to accurately identify "the sentinel margin," the point on a specimen where tumour lies closest to the resected edge in real-time during frozen section analysis.
\end{abstract}

\title{
Methods:
}

Eighteen patients with oral squamous cell carcinoma were enrolled into a prospective clinical trial and infused intravenously with 50mg of panitumumab-IRDye800CW 1-5 days prior to surgery. Resected specimens were imaged in a closed-field near-infrared optical imaging system in near-real time, and custom designed software was used to identify locations of highest fluorescence on deep and peripheral margins. The surgeon identified the sentinel margin blinded to optical specimen mapping, and then the regions of highest fluorescence were identified and marked for frozen analysis. Final pathology based on specimen reconstruction was used as reference standard.

\section{Results:}

Resected specimens were imaged in the operating room and fluorescence had a higher interobserver agreement with pathology (Cohen kappa value 0.96) than the surgeon (Cohen kappa values of 0.82 ) for the location of the closest margin. Plotting margin distance at the predicted sentinel margin location of each observer versus the actual closest margin distance at 
pathology demonstrated best correlation between fluorescence and pathology $\left(\mathrm{R}^{2}=0.98\right)$, with surgeon $\left(\mathrm{R}^{2}=0.75\right)$.

Principal conclusions:

Fluorescence imaging can improve identification of the sentinel margin in head and neck cancer resections, holding promise for rapid identification of positive margins and improved oncological outcomes.

Key words: Head and neck cancer, Oral squamous cell carcinoma, Tumour margins, Fluorescent image-guided surgery, Surgical oncology 


\section{INTRODUCTION}

In head and neck squamous cell carcinoma (HNSCC), surgery requires balancing complete tumour clearance with conservation of uninvolved tissue to preserve airway, voice and swallowing. Achieving adequate margins can be challenging (l) and it is established that positive margins are associated with local recurrence and decreased overall survival. Even with advances in operative technique and technology, positive margin rates have remained unchanged over the past 30 years $(2,3)$.

Assessment of tumour specimens to identify positive margins following removal allows surgeons to resect further tissue from the wound-bed if required to achieve adequate tumor clearance. This involves visual inspection and palpation of the specimen, with sampling of areas of concern from the specimen or wound bed for intraoperative frozen section analysis (FSA) (4). This process is inconsistent, surgeon dependent and complicated by sampling error in larger specimens.

Our group have previously demonstrated that ex vivo fluorescent imaging can identify the point on a specimen surface where tumour comes closest to the resection edge - the 'sentinel margin' $(5,6)$. The aim of the present study was to evaluate the accuracy and clinical value of fluorescent sentinel margin identification when compared prospectively to current clinical practice. We hypothesize that an objective, focused assessment of a specimen using optical imaging may reduce false negatives during intraoperative margin assessment, maintain orientation of sampled areas on the specimen to the wound bed and streamline the margin assessment process. 


\section{MATERIALS AND METHODS}

\section{Clinical Trial Design}

Between June 2018 and December 2019, patients with biopsy-proven primary or recurrent OSCC scheduled for curative surgery were enrolled into a prospective single centre, non-randomised, clinical trial evaluating a fluorescence molecular imaging agent called panitumumab-IRDye800CW. The institutional review board (IRB) of Stanford University approved this study and all subjects signed a written informed consent. The trial was approved by the research and the Food and Drug Administration (FDA), and registered with ClinicalTrials.gov (NCT03733210; NCT02415881; NCT03405142). This study was performed in accordance with the Helsinki Declaration of 1975 and its amendments, FDA's ICH-GCP guidelines and the laws and regulations of the United States.

\section{Study Workflow}

Enrolled patients were infused intravenously with 50mg of panitumumab-IRDye800CW 1-5 days prior to surgery(7). Dosing, timing and safety information on panitumumabIRDye800CW is provided in the Supplemental Information.

The study workflow is outlined in Figure 1. In the operating room, primary tumour specimens, as well as sampled margins for FSA, were imaged ex vivo on the back table immediately following removal from the patient in a closed-field near-infrared optical imaging system (IGP-ELVIS, LICOR Biosciences, Inc. Nebraska, US) (8). All tumour resections were $3 \mathrm{D}$ and were therefore repositioned within the IGP-ELVIS to capture each surface in a 2D plane. Imaged surfaces were denoted 'mucosal' if they primarily captured the mucosal aspect of the 
resection (Figure 1A). All other surfaces were denoted 'deep' (Figure 1B). Only imaged surfaces that required pathological evaluation by the surgical team were included in the study analysis.

Acquired images were exported as TIFF files from the optical imaging system to a laptop for near-real-time fluorescent analysis of the sentinel margin using ImageJ software (version 1.50i, NIH, Washington D.C, MD) as previously described $(5,6)$.

To compare our fluorescent analysis against current clinical practice, the board-certified surgeon, blinded to fluorescence, was asked to identify the point where tumour came closest to the specimen edge ex vivo. This was often done following at pathology in collaboration with the pathologist. Their specimen orientation and sentinel margin demarcation was photo and video documented. This photo was then used to compare the point of highest fluorescent signal on that surface by registering the two images against each other. A margin of error equivalent to one bread-loaf at pathology was used when comparing each prediction against final pathology as gold-standard. Individual bread loafs are approximately $5 \mathrm{~mm}$ thick (9), therefore if the surgeon or fluorescence is within $5 \mathrm{~mm}$ of the true sentinel margin, this would fall within the realms of "close enough" to ink, activating the pathologist to do selected radial margins in the area of concern. Where there was clinical concern that the specimen had close or positive margins, FSA was performed on the presumptive sentinel margins demarcated by the surgeon. These frozen sections were processed and analysed as standard-of-care and the results were reported to the operating team for intraoperative action as appropriate. Margins were defined in this study as "clear" if $\geq 5 \mathrm{~mm}$ from invasive tumour, close if $<5 \mathrm{~mm}$ from invasive tumour and positive if invasive carcinoma or carcinoma in situ was present at the specimen edge $(10,11)$. 
Primary tumour specimens underwent routine pathological processing and assessment, and all tissue cassettes were re-imaged in the IGP-ELVIS prior to paraffin embedding. A standard synoptic report was generated by a board-certified pathologist, which included identification of the final closest margin location and distance on mucosal and deep surfaces as seen on hematoxylin and eosin (H\&E) slides, serving as gold-standard to compare the sentinel margin predictions of the surgeon and fluorescence against. EGFR staining and expression quantification of selected slides was performed as previously described.(12)

To map all predicted sentinel margin locations against final pathology, locations were recorded categorically according to which pathology cassette they belonged to. Where two sentinel margin predictions were located on separate areas of a same cassette, they were differentiated with reference to their orientation on the cassette. A ruler was used to measure distance in millimetres from tumour edge (marked by the pathologist) to the specimen edge at each predicted sentinel margin on H\&E slides. Mean fluorescence intensity (MFI), defined as total counts per pixel area, divided by pixel area, was calculated for each acquired image of tissue taken intraoperatively for FSA using ImageStudio software (LI-COR Biosciences). MFI data was correlated to final tumour status (positive or negative).

\section{Statistical Analysis}

Scipy (version 1.4.1), Scikit-learn (version 0.22.2) and GraphPad Prism (version 8.0c) were used for statistical analysis. Cohen kappa (k) statistic was used to assess the strength of agreement of sentinel margin location of the two observers (surgeon and fluorescence) independently against gold-standard (final-pathology). Accuracy and error rate of sentinel margin location of each observer was calculated using standard statistics. The $95 \%$ confidence 
interval of each observer's accuracy was calculated based on a t-distribution. Accuracy p-values were calculated based on the number of cassettes for each specimen, with respect to random choices. Pearson and spearman correlation coefficients were calculated for margin distances at predicted sentinel margin locations of each observer against the actual closest margin distance at final pathology. A two-sided p-value of 0.05 or less was considered statistically significant.

\section{RESULTS}

\section{Observer Agreement and Accuracy in Sentinel Margin Prediction}

Primary tumour specimens from 18 patients were included in this study with 28 specimen surfaces imaged and analysed. The predicted sentinel margin location and corresponding measured margin distances at these locations per observer compared to final pathology is summarised in Table 1. Overall, areas of highest fluorescence strongly correlated final pathology (Cohen kappa 0.96). The surgeon had a relatively lower correlation with final pathology (Cohen kappa 0.81).

Accuracy of fluorescence intensity to identify the true sentinel margin was $96.4 \%$ (95\% CI $89.1-100, \mathrm{P}<0.001)$ compared to the surgeon at $82.1 \%(95 \% \mathrm{CI} 67.0-97.3, \mathrm{P}<0.001)$ as summarised in Table 2. Plotting margin distance at the predicted sentinel margin of each observer versus the actual closest margin distance at final pathology demonstrates better correlation between fluorescence and final pathology $\left(R^{2}=0.98,95 \%\right.$ CI $\left.0.93-1.00, P<0.001\right)$ compared to surgeon and final pathology $\left(\mathrm{R}^{2}=0.75,95 \% \mathrm{CI} 0.73-1.17, \mathrm{P}<0.001\right)$ (Figure 2). 


\section{Clinical Value of Fluorescence in Sentinel Margin Analysis}

The surgeon's prediction of the sentinel margin disagreed with final pathology in 4 out of 28 cases compared to 1 out of 28 cases with fluorescence. In the one instance where fluorescence disagreed with final pathology, the fluorescent predicted sentinel margin location was in concordance with the surgeon's prediction. This equates to a clinically significant improvement in intraoperative frozen section sampling in 3/28 (10.7\%) surfaces analysed with the use of fluorescence over current standard of care.

Closer examination of the one case where there was tumor close to the mucosal surface that was not identified by fluorescence as being the area of highest signal, revealed that this tumour was in fact a small secondary focus (Figure 3). IHC staining and EGFR quantification of regions where each focus of tumour came closest to the peripheral margin demonstrated EGFR expression at both points, but with higher EGFR expression (81.2\%) where there was macroscopically visible disease on the main tumour focus versus $(43.2 \%)$ on the smaller secondary focus (Figure 4).

In three cases where the fluorescent sentinel margin prediction outperformed conventional palpation by the surgeon, the final margin was close $(<5 \mathrm{~mm})$ in all cases, as summarized in Figure 5. 


\section{DISCUSSION}

This prospective study compares fluorescence with current intraoperative margin assessment, evaluated against final pathology as gold-standard. It builds on previous retrospective studies in which we developed the concept of sentinel margin detection using relative fluorescence intensities and showed that margin distance inversely correlates with areas of highest fluorescent intensity on the deep and the mucosal surfaces $(5,6)$. These studies laid the foundation for the current prospective trial, but were limited by their retrospective nature, which meant that only specimens where measurable bread loafs coincided with the intensity peaks on the deep surface, or areas of highest fluorescence intensity on the peripheral surface, could be included for analysis. Tissue is relatively homogenous, thereby disrupting optical properties and enabling this technique to work consistently across specimens. Furthermore, although EGFR is heterogenous across the different patient cancer specimens, it is high enough to allow fluorescence to penetrate through the tissue margin. This study, unlike previous research in this technique is a prospective comparison to the surgeon.

This study demonstrates that ex vivo fluorescent molecular imaging of head and neck resections can improve objective detection of tumour that comes closest to the specimen edge when compared to standard of care, which was exemplified in $11 \%$ of cases where fluorescencebased sentinel margin assessment outperformed standard of care pathological assessment by the surgeon, suggesting this approach has potential to improve positive margin rate outcomes. Accurate isolation of the sentinel margin in the operating room within minutes has several benefits. Of highest clinical priority, it holds promise to minimize false negative intraoperative margin assessment (13). From a resource utilisation standpoint, reduced tissue sampling and 
reduced FSA streamlines operative workflow and decreases pathology demand $(14,15)$. By identifying the sentinel margin in the operating room immediately following resection, there is promise for improved in vivo and ex vivo orientation of the specimen to the wound bed, which could increase precision during wound bed re-resection, although this was not assessed here and requires focused evaluation in future studies $(16,17)$. Anecdotally in this study, fluorescence imaging followed by sentinel margin analysis took approximately 5 to 10 minutes to complete (depending on the complexity of the specimen and how many surfaces were being imaged) whereas frozen sectioning typically takes 20-30 minutes per FSA. Hence, additional benefit can be found in providing directional information to the pathologist for areas to be assessed by FSA.

All 18 HNSCC specimens in this study originated from the oral cavity, but resection units varied, including glossectomies, maxillectomies, mandibulectomies and composite resections. Our findings demonstrate that fluorescence molecular imaging of complex 3D resections involving soft tissue and bone is feasible and this is a significant strength of this study, as current ex vivo margin analysis in these complex specimens is particularly difficult (18). It is in these resections that there is a short window-of-opportunity for surgeon orientation of specimen to wound bed, and where communication of margin locations between surgeon and pathologist are prone to error (19).

Prior to undertaking this study, we hypothesized that specimen positioning and intratumoral heterogeneity of EGFR could impact accuracy of fluorescent sentinel margin identification. In this study we identified a case where a smaller tumour satellite with both an associated lower EGFR expression and fluorescence intensity, resulted in a false negative result because it lay closer to the resection edge than the main tumour focus. Based on this, and in line 
with the results of our previous studies, we would advise that at least 2-3 regions of highest fluorescent signal on a specimen surface be sampled (6). Sampling the three areas of highest fluorescence on the peripheral margin would have picked up this separate focus of microscopic disease that was not visually detectable or palpable, consistent with our previous studies (7).

In one of the three cases where fluorescence was superior to surgeon sentinel margin prediction, the standard approach still identified a close margin of $2 \mathrm{~mm}$. This highlights an important clinically relevant point, that there may be more than one location on a specimen surface, and multiple surfaces on one specimen, where tumour comes close to the resection edge. It is precisely in these cases where fluorescence can be of significant added value, as re-resection from the wound-bed at multiple locations may be necessary and without fluorescence guidance may be missed. The specimen imaging techniques are agnostic to the fluorescent agent used and may thus also apply beyond paniatumumab-IRDye800CW (20), (21) (22). It is worth mentioning that non-fluorescent optical dyes have been investigated for margin analysis with promising results $(23,24)(25)$.

There are study limitations worth highlighting. Firstly, where a specimen surface that the surgeon and/or pathologist were not concerned for close margins, fluorescence analysis and FSA were not performed to reduce intraoperative pathology time and allow focus on clinically relevant margin assessment, consistent with the philosophy that margin assessment strategy should be used for clinical guidance only. Moreover, we did not examine margin distances at high fluorescent areas on specimen surfaces where there was no clinical suspicion for close margins. As such, we cannot comment on the accuracy of fluorescent imaging at identifying the sentinel margin in all surfaces of all specimens resected in this study. Future trials could 
circumnavigate the issue of intraoperative delay, by prospectively collecting tissue samples at pathology of the identified sentinel margin location on all surfaces of all specimens (based on both fluorescent analysis and surgeon prediction) and then retrospectively analyse the margin distances on these tissue samples later. Secondly, fluorescence margin analysis in this study was performed by one person. While this kept variability in fluorescent margin analysis and interpretation constant, a learning curve for performing the analysis and discrepancies in analysis between users was not examined. With increasing adoption of artificial intelligence technology in surgery, it is foreseeable that future use of this strategy may move toward computer automated techniques.

\section{CONCLUSION}

Image-guided analysis of specimens can improve identification of the true closest margin in 3D resections with potential to reduce positive margin rates. Results of the current study determined a clinically significant value of fluorescence-based margin assessment in $11 \%$ of patients. A study examining the value of this technique in improving communication of margin location between surgeon and pathologist as well as evaluating the efficacy of surgical guided reresection should follow. 


\section{FINANCIAL DISCLOSURE}

This work was supported in part by the Stanford Comprehensive Cancer Center, the Stanford University School of Medicine Medical Scholars Program, the Garnett Passe and Rodney William's Memorial Foundation, the Fulbright Association, the Netherlands Organization for Scientific Research (Rubicon; 019.171LW.022), the National Institutes of Health and the National Cancer Institute (R01CA190306), and an institutional equipment loan from LI-COR Biosciences, Inc.

\section{DISCLAIMER}

E.L.R. served as a consultant for LI-COR Biosciences Inc. and has equipment loans from this company. All other authors declare no conflict of interest. 


\section{KEY POINTS}

Question: How does ex vivo fluorescent molecular imaging of head and neck cancer resections compare with current standard of care in identifying the true closest margin?

Pertinent findings: In this prospective observational clinical trial including 18 consecutive patients who underwent head and neck cancer surgical resections, fluorescence-based sentinel margin assessment outperformed the surgeon at identifying the true closest margin at final pathology in $11 \%$ of cases. Fluorescence had a higher interobserver agreement with final pathology (Cohen kappa value 0.96) than the surgeon (Cohen kappa values of 0.82) and plotting the margin distance at the predicted sentinel margin location of each observer versus the actual closest margin distance at final pathology demonstrated best correlation between fluorescence and final pathology $\left(\mathrm{R}^{2}=0.98\right)$.

Implications for patient care: Broad translation of an ex vivo fluorescent molecular imagebased approach to sentinel margin identification could improve accuracy of intraoperative margin sampling, with overall potential to reduce positive margin rates in surgery. 


\section{REFERENCES}

1. Luryi AL, Chen MM, Mehra S, Roman SA, Sosa JA, Judson BL. Positive surgical margins in early stage oral cavity cancer: an analysis of 20,602 cases. Otolaryngol Head Neck Surg. 2014;151:984-990.

2. Smits RW, Koljenović S, Hardillo JA, et al. Resection margins in oral cancer surgery: Room for improvement. Head Neck. 2016;38 Suppl 1:E2197-2203.

3. Orosco RK, Tapia VJ, Califano JA, et al. Positive surgical margins in the 10 most common solid cancers. Sci Rep. 2018;8:5686.

4. Kain JJ, Birkeland AC, Udayakumar N, et al. Surgical margins in oral cavity squamous cell carcinoma: Current practices and future directions. Laryngoscope. 2020;130:128-138.

5. van Keulen S, Nishio N, Birkeland A, et al. The Sentinel Margin: Intraoperative ex vivo specimen mapping using relative fluorescence intensity. Clin Cancer Res. 2019;25:4656-4662.

6. Fakurnejad S, Krishnan G, van Keulen S, et al. Intraoperative molecular imaging for ex vivo assessment of peripheral margins in oral squamous cell carcinoma. Front Oncol. 2019;9:1476. 
7. Nishio N, van den Berg NS, van Keulen S, et al. Optimal dosing strategy for fluorescence-guided surgery with panitumumab-irdye $800 \mathrm{cw}$ in head and neck cancer. Mol Imaging Biol. 2020;22:156-164.

8. van Keulen S, van den Berg NS, Nishio N, et al. Rapid, non-invasive fluorescence margin assessment: Optical specimen mapping in oral squamous cell carcinoma. Oral Oncol. 2019;88:58-65.

9. Williams MD. Determining adequate margins in head and neck cancers: practice and continued challenges. Curr Oncol Rep. 2016;18:54.

10. Alicandri-Ciufelli M, Bonali M, Piccinini A, et al. Surgical margins in head and neck squamous cell carcinoma: what is 'close'? Eur Arch Otorhinolaryngol. 2013;270:2603-2609.

11. Colevas AD, Sue SY, David GP, et al. NCCN guidelines insights: Head and neck cancers, Version 1.2018. Journal of the National Comprehensive Cancer Network J Natl Compr Canc Netw. 2018;16:479-490.

12. Zhou Q, van den Berg NS, Rosenthal EL, et al. EGFR-targeted intraoperative fluorescence imaging detects high-grade glioma with panitumumab-IRDye800 in a phase 1 clinical trial. Theranostics. 2021;11:7130-7143. 
13. Maxwell JH, Thompson LD, Brandwein-Gensler MS, et al. Early oral tongue squamous cell carcinoma: Sampling of margins from tumor bed and worse local control. JAMA Otolaryngol Head Neck Surg. 2015;141:1104-1110.

14. Kubik MW, Sridharan S, Varvares MA, et al. Intraoperative margin assessment in head and neck cancer: A case of misuse and abuse? Head Neck Pathol. 2020;14:291-302.

15. DiNardo LJ, Lin J, Karageorge LS, Powers CN. Accuracy, utility, and cost of frozen section margins in head and neck cancer surgery. Laryngoscope. 2000;110:1773-1776.

16. Kerawala CJ, Ong TK. Relocating the site of frozen sections--is there room for improvement? Head Neck. 2001;23:230-232.

17. van Lanschot CGF, Mast H, Hardillo JA, et al. Relocation of inadequate resection margins in the wound bed during oral cavity oncological surgery: A feasibility study. Head Neck. 2019;41:2159-2166.

18. Lubek JE, Magliocca KR. Evaluation of the bone margin in oral squamous cell carcinoma. Oral Maxillofac Surg Clin North Am. 2017;29:281-292.

19. Weinstock YE, Alava I, 3rd, Dierks EJ. Pitfalls in determining head and neck surgical margins. Oral Maxillofac Surg Clin North Am. 2014;26:151-162. 
20. Mizushima T, Ohnishi S, Shimizu Y, et al. Fluorescent imaging of superficial head and neck squamous cell carcinoma using a $\gamma$-glutamyltranspeptidase-activated targeting agent: a pilot study. BMC Cancer. 2016;16:411.

21. Pan J, Deng H, Hu S, et al. Real-time surveillance of surgical margins via ICG-based near-infrared fluorescence imaging in patients with OSCC. World J Surg Oncol. 2020;18:96.

22. Christensen A, Juhl K, Persson M, et al. uPAR-targeted optical near-infrared (NIR) fluorescence imaging and PET for image-guided surgery in head and neck cancer: proof-ofconcept in orthotopic xenograft model. Oncotarget. 2017;8:15407-15419.

23. McCaul JA, Cymerman JA, Hislop S, et al. LIHNCS - Lugol's iodine in head and neck cancer surgery: a multicentre, randomised controlled trial assessing the effectiveness of Lugol's iodine to assist excision of moderate dysplasia, severe dysplasia and carcinoma in situ at mucosal resection margins of oral and oropharyngeal squamous cell carcinoma: study protocol for a randomised controlled trial. Trials. 2013;14:310.

24. Petruzzi M, Lucchese A, Baldoni E, Grassi FR, Serpico R. Use of Lugol's iodine in oral cancer diagnosis: an overview. Oral Oncol. 2010;46:811-813.

25. Algadi HH, Abou-Bakr AA, Jamali OM, Fathy LM. Toluidine blue versus frozen section for assessment of mucosal tumor margins in oral squamous cell carcinoma. BMC Cancer. 2020;20:1147. 


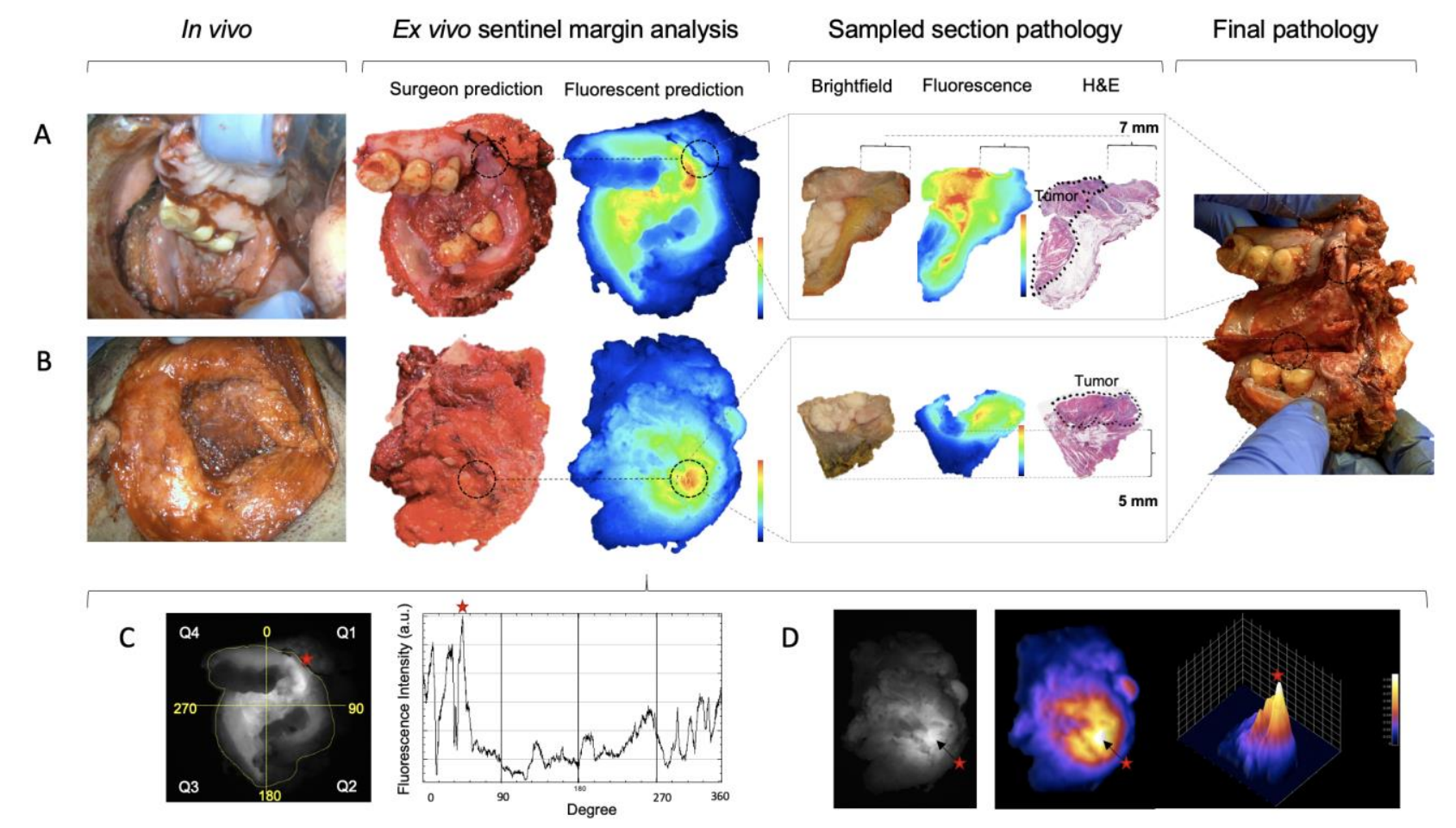

FIGURE 1. Study workflow demonstrated in representative patient 18 with a right sided retromolar trigone squamous cell carcinoma. The patient was infused intravenously with 50mg of Panitumumab-IRDye800CW three days prior to surgery. From left to right, the workflow shows the lesion in vivo and then ex vivo, examining the (A) mucosal, and (B) deep surfaces of the resection. On both surfaces the surgeon and fluorescence were in agreement as to the location of the sentinel margin. Sampled tissue from these locations underwent closed-field fluorescent imaging and then H\&E staining to evaluate the margin distance. Following this the specimen was examined at final pathology. C. Mucosal surface fluorescent sentinel margin analysis in this patient as demonstrated by a mask manually fitted around the periphery of the specimen within $1 \mathrm{~mm}$ of the resection edge followed by a graph showing the raw fluorescence data of each point along the mask in an 8-bit grayscale format enabling isolation of the area of highest fluorescence on the periphery (represented by the red star). D. Deep surface fluorescent sentinel margin analysis as demonstrated by using the 3D signal-mapping tool to scale and isolate the area of highest fluorescence intensity (represented by the red star). 
Surgeon

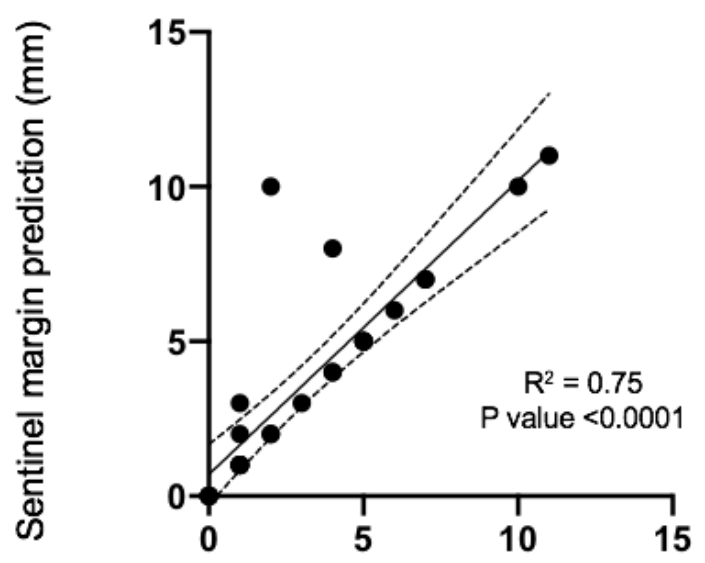

Fluorescence

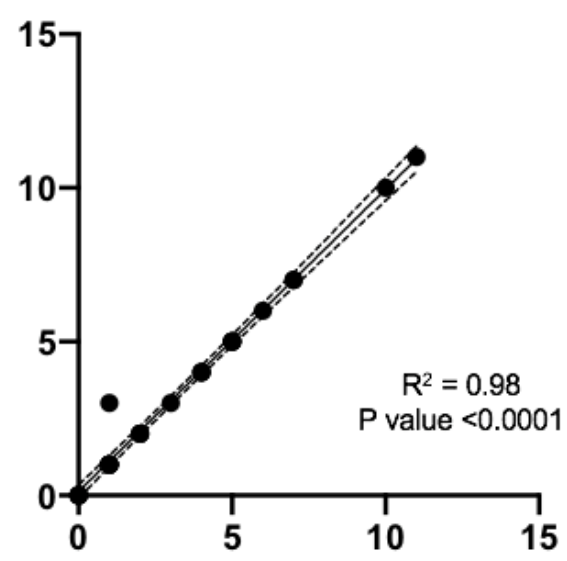

Closest margin at final pathology $(\mathrm{mm})$

FIGURE 2. Correlation between distance at predicted sentinel margin by observer with distance of closest margin on final pathology. 


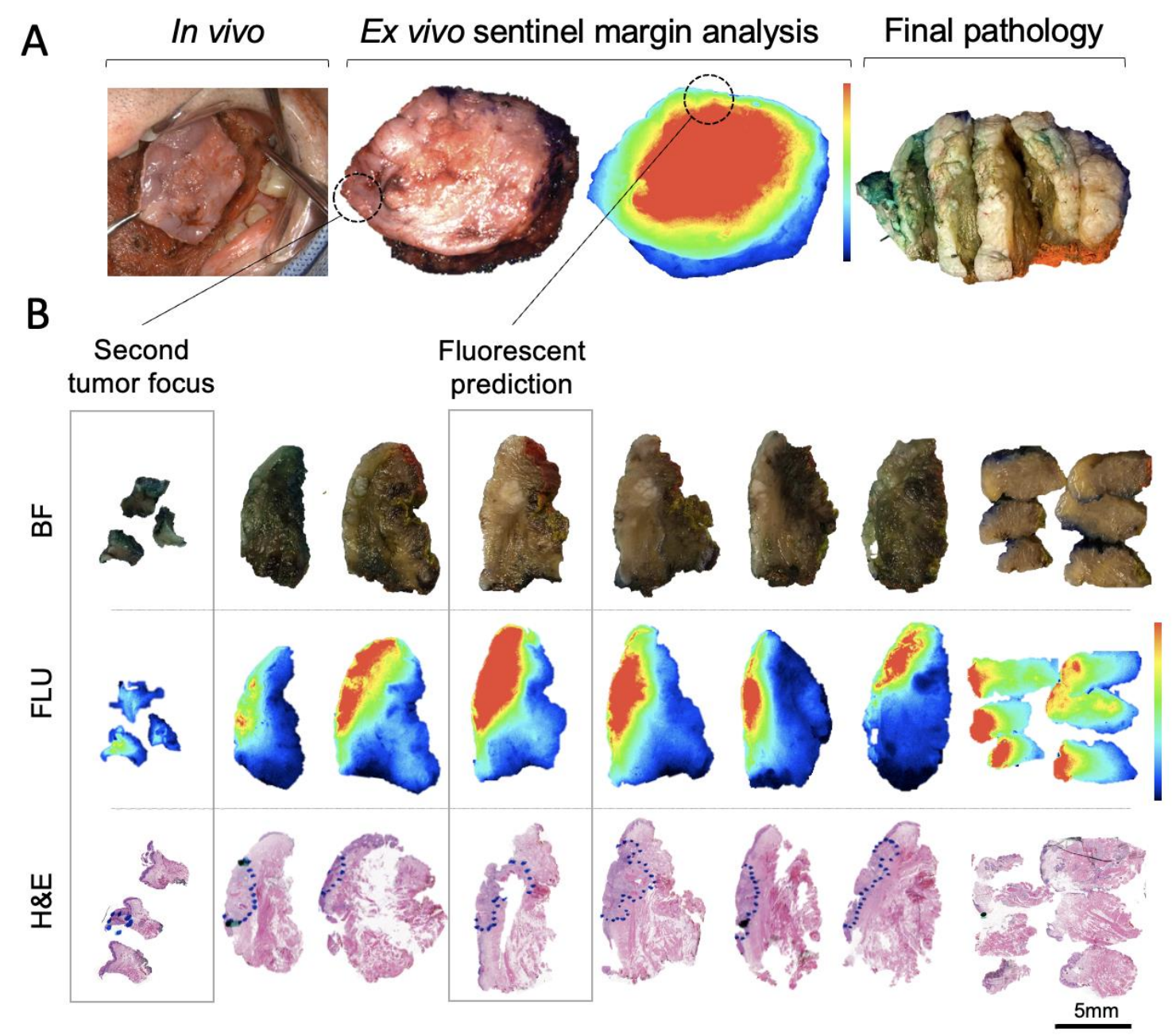

FIGURE 3. A. Specimen evaluation workflow demonstrating the partial glossectomy resection in situ, the ex vivo specimen imaged in brightfield and closed-field fluorescence, followed by a depiction of the specimen bread loafed at final pathology. B. The main centrally located tumor came closest to the specimen edge superiorly, at a point accurately identified by both fluorescence and the surgeon to be the closest margin for this tumour $(3 \mathrm{~mm})$. The smaller separate focus of tumor was not macroscopically visible and registered as the third highest region of fluorescent signal on the peripheral margin. (Blue dots outline tumour boundaries on the mucosal surface).

BF: Brightfield, FLU: Fluorescence, H\&E: Haematoxylin and eosin. 

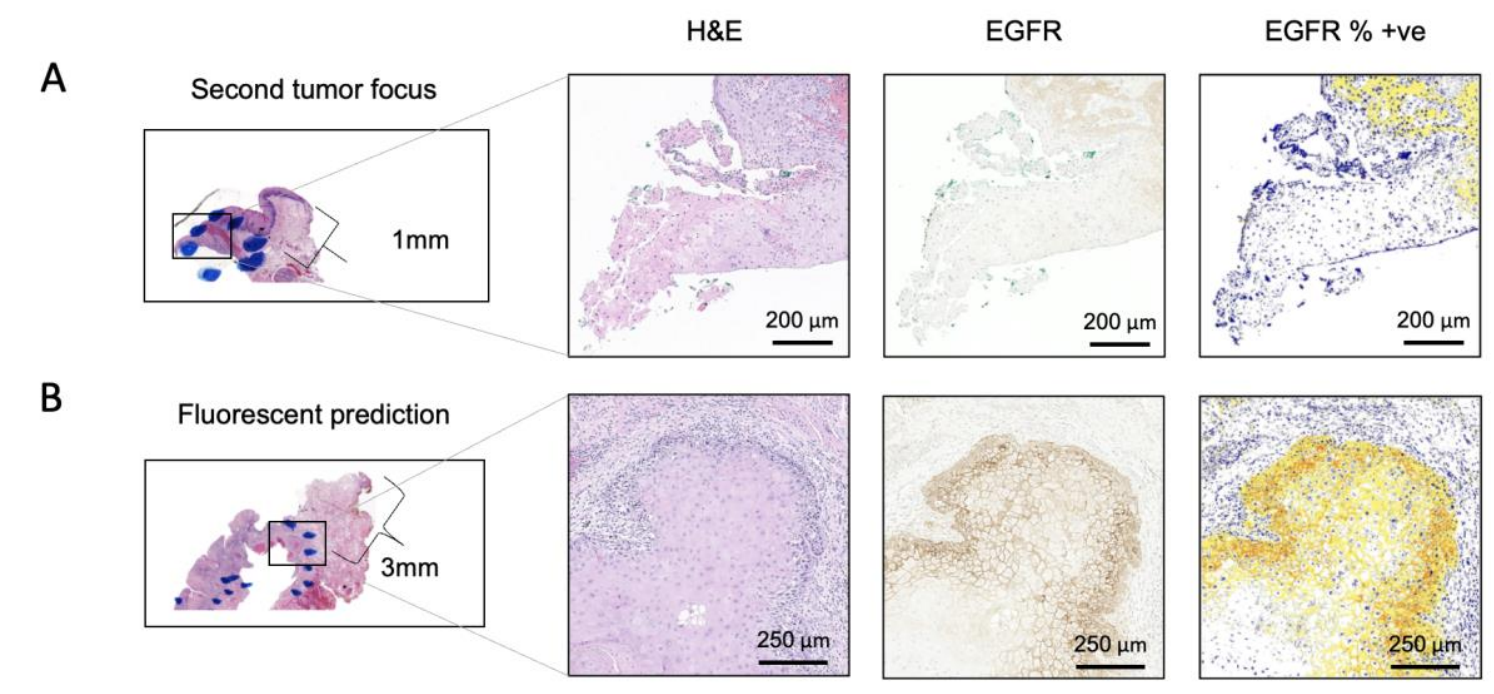

strong +ve medium +ve weak +ve negative

FIGURE 4. A. Magnified view of two bread loafs demonstrating margin distance of $3 \mathrm{~mm}$, where surgeon and fluorescence predicted the sentinel margin on the main tumor, compared to a margin distance of $1 \mathrm{~mm}$ where a the sperate secondary focus of tumor came close to the anterior specimen edge. B. Slides confirming the presence of SCC and EGFR expression at the point on each tumor focus closes to the margin edge. In the main tumour, EGFR expression was quantified at $81.2 \%$ compared to $43.2 \%$ at the secondary focus.

H\&E: Haematoxylin and eosin, EGFR: Epithelial growth factor receptor, +ve: Positive. 


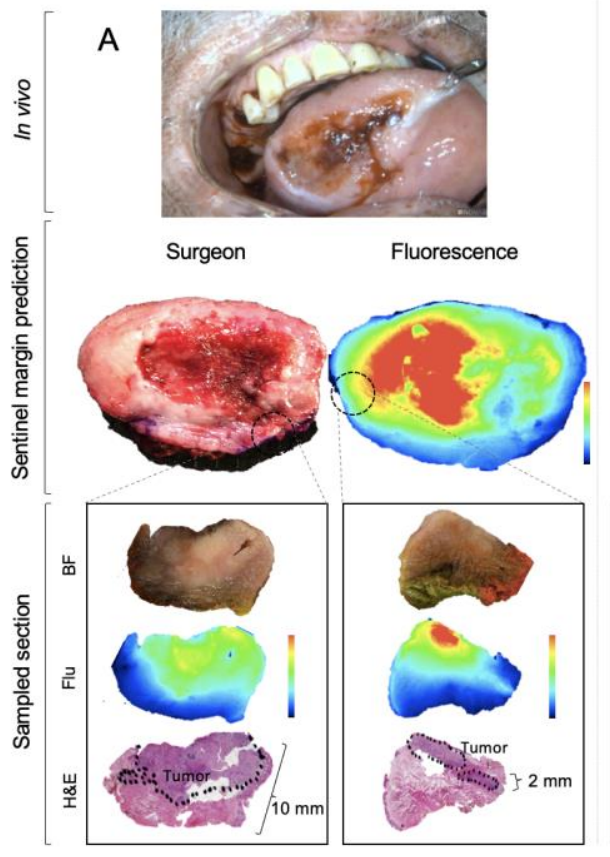

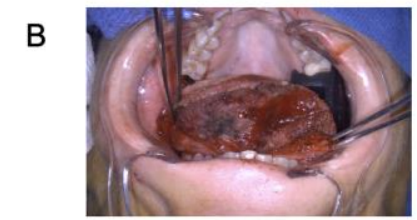
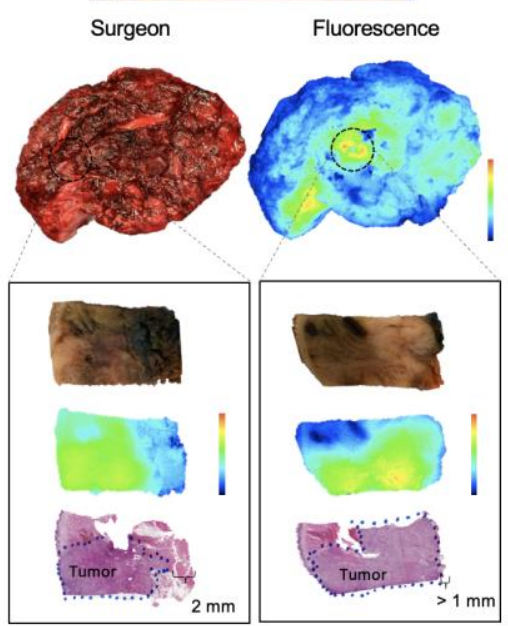

\section{C}
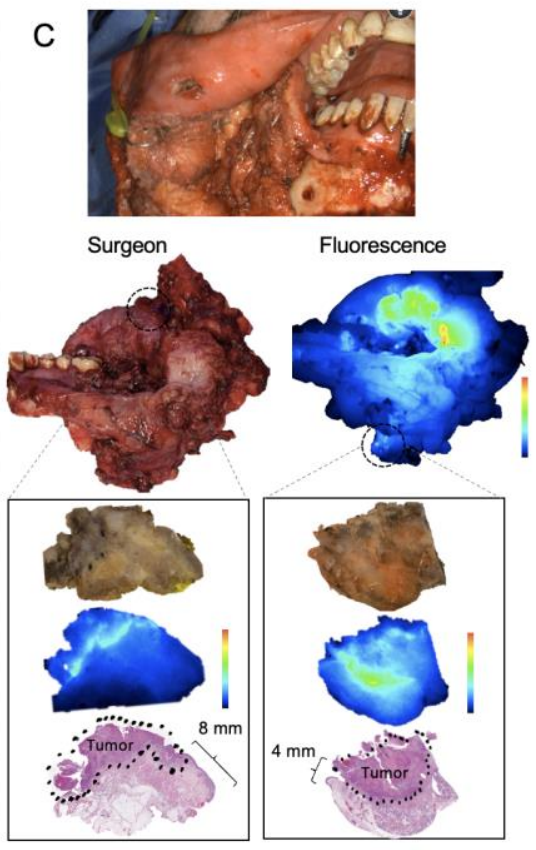

FIGURE 5. Cases where fluorescence sentinel margin identification demonstrated improved identification of the true closest margin when compared to conventional analysis technique by the surgeon. A. Patient 1 with a pT3 right lateral tongue SCC infused 2 days prior to surgery. B. Patient 2 with pT4a right lateral tongue SCC infused 1 day prior to surgery. C. Patient13 with a pT4a right retromolar SCC infused 2 days prior to surgery.

Flu: Fluorescence, BF: Brightfield, H\&E: Haematoxylin and eosin 


\section{TABLES}

Table 1. Summary of Interobserver prediction of sentinel margin location and corresponding margin distance versus actual closest margin location and distance at pathology

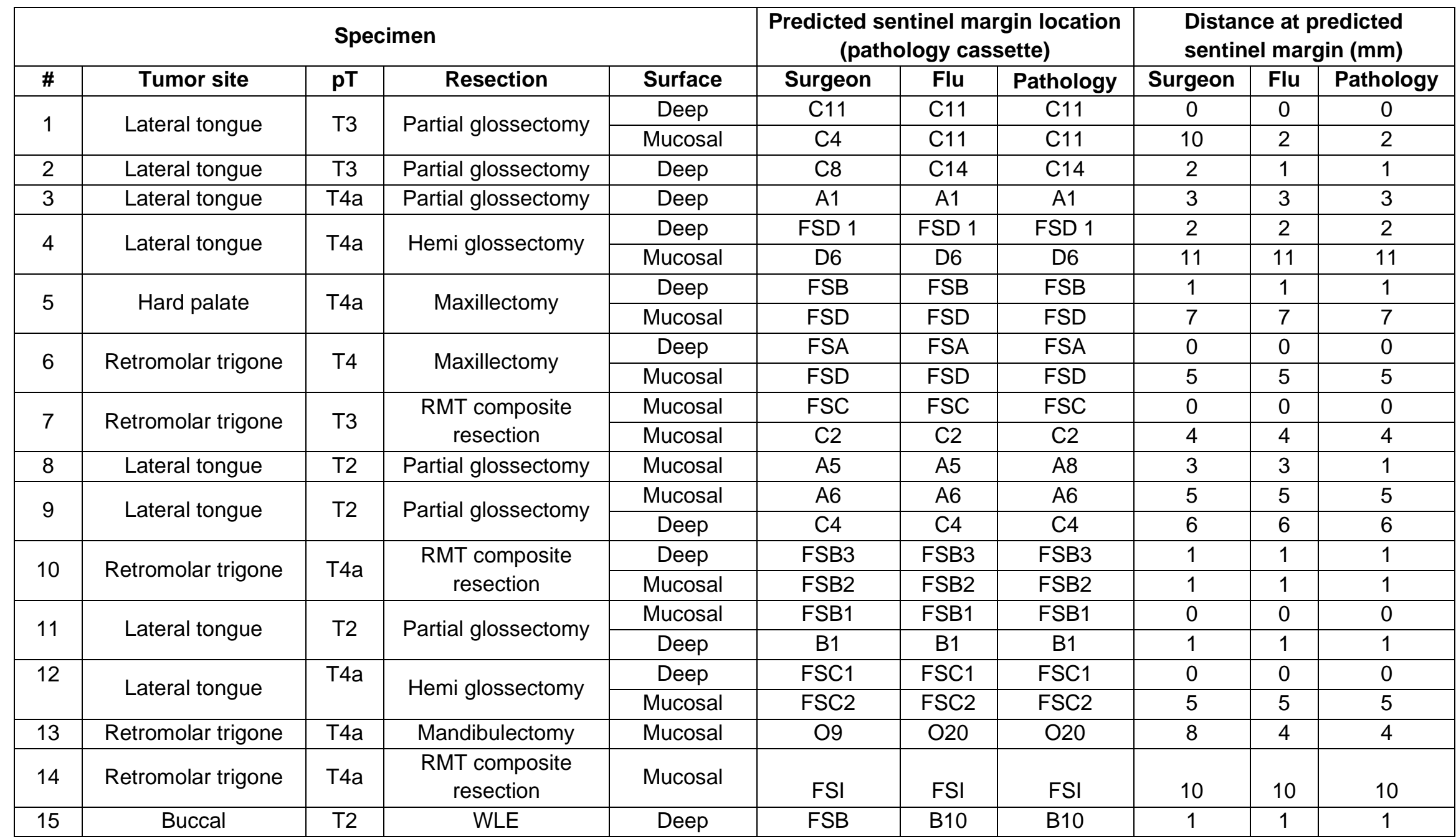




\begin{tabular}{|c|c|c|c|c|c|c|c|c|c|c|}
\hline 16 & Alveolar ridge & $\mathrm{T} 2$ & Mandibulectomy & Mucosal & $\mathrm{D} 8$ & D8 & D8 & 4 & 4 & 4 \\
\hline 16 & Buccal & $\mathrm{T} 2$ & WLE & Mucosal & FSA & FSA & FSA & 2 & 2 & 2 \\
\hline \multirow{2}{*}{18} & \multirow{2}{*}{ Retromolar trigone } & \multirow{2}{*}{ T3 } & \multirow{2}{*}{$\begin{array}{l}\text { RMT composite } \\
\text { resection }\end{array}$} & Mucosal & B11 & B11 & B11 & 7 & 7 & 7 \\
\hline & & & & Deep & B16 & B16 & $\mathrm{B} 16$ & 5 & 5 & 5 \\
\hline
\end{tabular}

pT: pathological T-stage, RMT: Retromolar trigone, Flu: Fluorescence 
Table 2. Accuracy of observer at identifying the final closest margin location

\begin{tabular}{|l|l|l|l|l|l|}
\hline & $\begin{array}{l}\text { Interobserver } \\
\text { agreement with } \\
\text { final pathology } \\
(\text { Cohen kappa) }\end{array}$ & Accuracy (\%) & $\begin{array}{l}\text { 95\% confidence } \\
\text { interval }\end{array}$ & Error rate (\%) & P value \\
\hline Surgeon & 0.82 & 0.64 & $(0.45,0.83)$ & 17.9 & $<0.001$ \\
\hline Fluorescence & 0.96 & 0.86 & $(0.72,1.00)$ & 3.6 & $<0.001$ \\
\hline
\end{tabular}




\section{GRAPHICAL ABSTRACT}
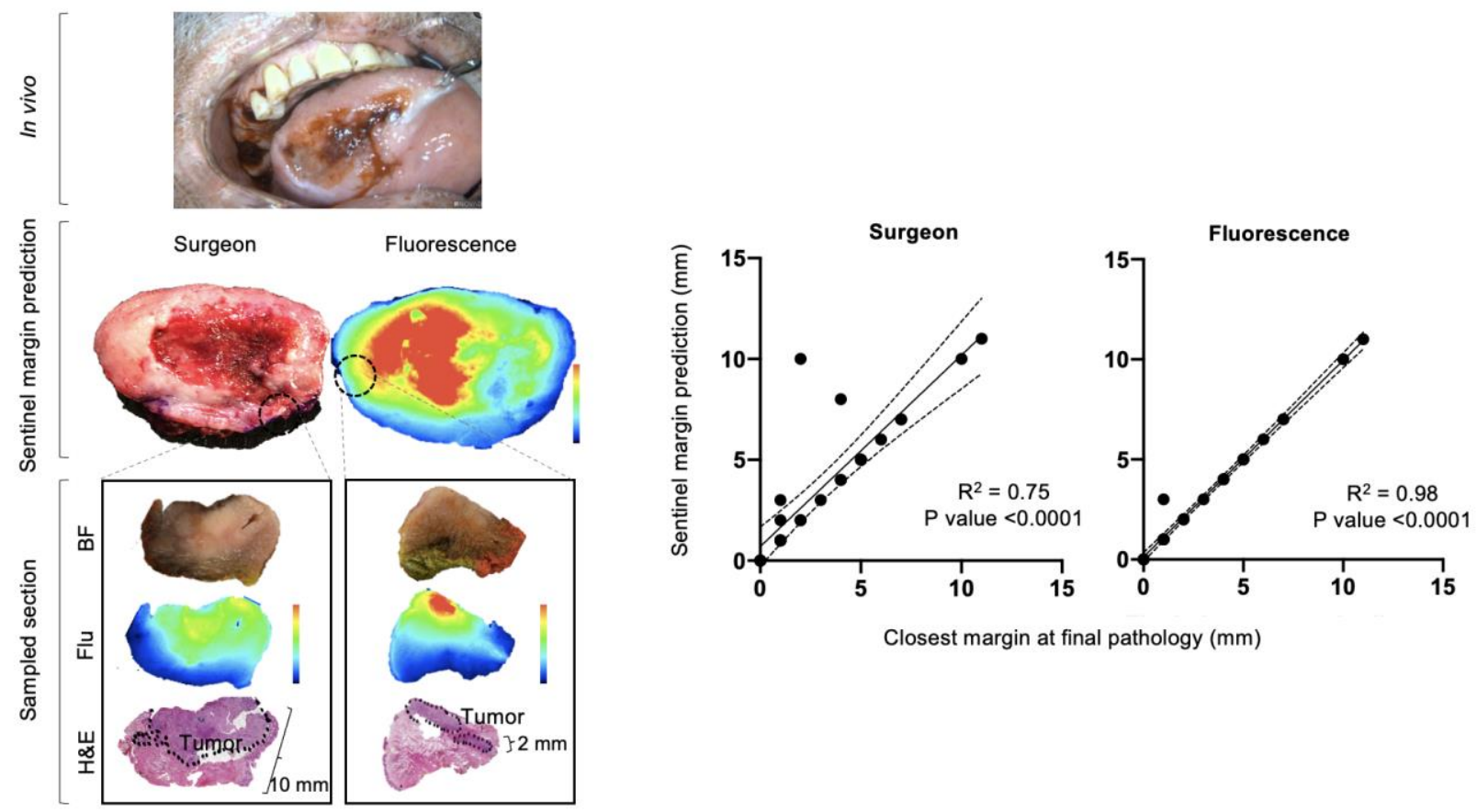